\title{
Investigation of the Effects of Continuous Low- Dose Epidural Analgesia on the Autonomic Nervous System Using Hilbert Huang Transform
}

\author{
Wei-Ren Chuang ${ }^{1}$, Jia-Rong Yeh${ }^{1}$, Li-Kuei Chen², \\ Yin-Yi Han ${ }^{2}$ and Jiann-Shing Shieh ${ }^{1}$ \\ ${ }^{1}$ Dept. of Mechanical Engineering, Yuan Ze University \\ ${ }^{2}$ Dept. of Anesthesiology, College of Medicine, National Taiwan University
}

\begin{abstract}
Effects of continuous low-dose epidural bupivacaine (0.05-0.1\%) infusion on the Doppler velocimetry for labor analgesia have been well documented. The aim of this study was to monitor the activity of the autonomic nervous system (ANS) for women in labor based on Hilbert Huang transform (HHT), which performs signal processing for nonlinear systems, such as human cardiac systems. Thirteen pregnant women were included in the experimental group for labor analgesia. They received continuous epidural bupivacaine $0.075 \%$ infusion. The normal-tonormal intervals (NN-interval) were downloaded from an ECG holter. Another 20 pregnant women in non-anesthesia labor (average gestation age was 38.6 weeks) were included in the comparison group. In this study, HHT was used to decompose components of ECG signals, which reflect three different frequency bands of a person's heart rate spectrum (viz. high frequency (HF), low frequency (LF) and very low frequency (VLF)). It was found that the change of energy in subjects without anesthesia was more active than that with continuous epidural bupivacaine $0.075 \%$ infusion. The energy values of the experimental group (i.e., labor analgesia) of HF and LF of ANS activities were significantly lower $(P<0.05)$ than the values of the comparison group (viz. labor without analgesia), but the trend of energy ratio of LF/HF was opposite. In conclusion, the sympathetic and parasympathetic components of ANS are all suppressed by continuous lowdose epidural bupivacaine $0.075 \%$ infusion, but parasympathetic power is suppressed more than sympathetic power.
\end{abstract}

Keywords: Epidural infusion, labor analgesia, autonomic nervous system, ECG holter, Hilbert Huang transform, heart rate spectrum, sympathetic, parasympathetic.

\section{INTRODUCTION}

Epidural analgesia is commonly used for pain relief during labor in modern obstetric practice, but the influence on the fetus and childbirth are still called into question. Epidural analgesia can prolong the course of labor and increase the rate of instrument delivery. The effects of continuous epidural analgesia on Doppler velocimetry of uterine arteries during labor analgesia have been reported [1] showing that the velocimet-

Corresponding author: Prof. Jiann-Shing Shieh, PhD, Department of Mechanical Engineering, Yuan Ze University, 135 Yuan-Tung Rd., Chung-Li, Taoyuan, 320, Taiwan. Tel: 886-3-4638800 ext. 2470. email: jsshieh@saturn.yzu.edu.tw 
ric indices of uterine vascular resistance significantly increased 1, 2, and 4 hours after epidural infusion, and then returned to the baseline (before analgesia) after delivery.

Previous studies have shown that the autonomic nervous system (ANS) contributes to the regulation of heart contraction on both beating rate and contracting volume in patients [2]. In 1981, Akselrod et al. [3] found that an ECG could present activity of ANS via the Fourier spectrum by Fast Fourier Transform (FFT). Thereafter, the reactions of different frequency bands of ANS were investigated by many different methods. They reflected the reactions of parasympathetic tone via the high-frequency (HF) band $(0.15 \sim 0.4 \mathrm{~Hz}$ for human) of energy of the heart beat spectrum and the reactions of sympathetic tone via low-frequency (LF) band (0.04 0.15 Hz for human) of energy of the heart beat spectrum [4]. Sympathovagal effects and the ANS response were assessed in women in labor under epidural or spinal anesthesia, which can be treated by heart rate variability analysis $[2,5,6]$. However, traditional assessments of ANS activity and sympathovagal effects are based on the assumption of linearity, while human cardiac system performs as a nonlinear system. This work presents a new assessment of ANS activity based on an innovative technique for a nonlinear system.

Empirical mode decomposition (EMD) proposed by Dr. Huang in 1998 is an adaptive signal processing and analysis algorithm for nonlinear and non-stationary systems [8]. It is an innovative method with an advantage over the FFT which can only handle linear systems. Moreover, the decomposed components, named intrinsic mode frequency (IMF), can be used to derive the time-frequency-amplitude distribution using Hilbert transform [9]. The combination of EMD and Hilbert transform is named Hilbert Huang transform (HHT) [8].

In this study, 13 heart rate recordings during the labor processes and 20 subjects of a control group were decomposed into the first 8 IMFs by EMD. Time-frequencyamplitude distributions were then derived from the IMFs using Hilbert transform. The connections between these particular IMFs and sympathetic or parasympathetic tone were verified via the averaged periods of IMFs and the frequency bands of HHT. HF and LF bands of the heart beat spectrum were evaluated using the energy density of the correlated IMFs decomposed from heart beat time series. Therefore, the reaction on ANS caused by epidural analgesia could be estimated via the total energy of the special frequency band of heart beat spectrum. The aim of this study was to show how continuous low-dose epidural bupivacaine infusion affected the activity of ANS for women in labor compared with subjects without analgesia.

\section{HILBERT-HUANG TRANSFORM APPLIED IN CONTINUOUS EPIDURAL ANALGESIA}

The Hilbert-Huang transform is a combination of empirical mode decomposition (EMD) and Hilbert transform to decompose a signal into a limited number of intrinsic mode functions (IMF), and to derive the instantaneous time-frequency-amplitude distribution of each IMF. Figure 1 shows an illustration of HHT and more details are provided below. The core of HHT is the EMD shown inside the dashed rectangle. The output of IMFs was used to generate Hilbert spectrum of signal by Hilbert transform. A sifting process is the main process of EMD. A signal, $s(t)$, was decomposed by the sifting process to gain a candidate for IMF. The IMF candidate must satisfy two necessary 


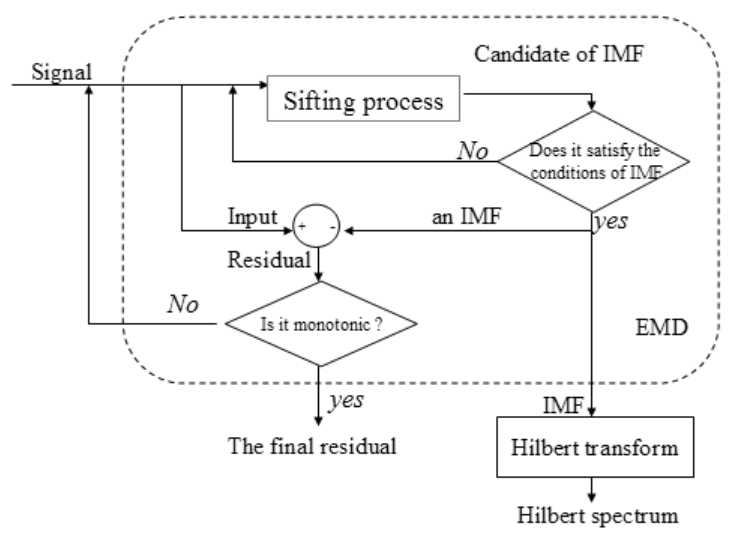

Figure 1. Illustration of Hilbert Huang Transform

conditions to be identified as a formal IMF, and it is processed again and again until it does. These two necessary conditions are:

1) In the whole data set, the number of extrema and the number of zero-crossings must either equal or differ at most by one.

2) At any point, the mean value of the envelope defined by the local maxima and the envelope defined by the local minima is zero.

Sifting is a process in which a candidate of IMF is the riding fluctuation on the signal trend. This trend is defined as the mean of envelopes (i.e., upper and lower envelopes). The upper envelope is developed by connecting the local maxima using cubic spline. Similarly, the lower envelope is obtained via local minima. Therefore, a sifting process is built up in the following steps:

Step 1: Use the original data, $s(t)$, for the decomposition for the first IMF, and use the residual, $r(t)$, of the last decomposition to compute the remainder IMFs. Connect all the local maxima using cubic spline to develop the upper envelope. Repeat the procedure to the local minima to produce the lower envelope.

Step 2: Obtain the local trend of data, $m_{l}(t)$, using the mean of upper and lower envelopes.

Step 3: The difference between data and its local trend is the candidate of IMF and noted as $h_{l}(t)$. The relationship between $h_{l}(t)$ and $m_{l}(t)$ can be expressed as: $h_{l}(t)=s(t)-m_{l}(t)$.

Step 4: Ideally, $h_{l}(t)$ should satisfy these two necessary conditions for an IMF. If not, repeat step 1 to step 3 using $h_{l}(t)$ as the data to generate the next candidate of IMF until the candidate satisfies the necessary conditions. A candidate, which satisfies these conditions, can be identified as an IMF noted as $c_{1}(t)$, and then a decomposing process is completed. 
Step 5: The residual of a decomposing process is obtained by the following equation:

$$
r_{l}(t)=s(t)-c_{1}(t)
$$

Step 6: Use $r_{l}(t)$ as the new data and repeat step 1 through step 5 to obtain the next IMF $c_{i}(t)$ and residual $r_{i}(t)$. The decomposing process is repeated until the residual is a monotonic function.

The original signal $s(t)$ can be represented by a combination of a number of IMF components and the final residual of decomposition is shown in the following eqaution:

$$
s(t)=\sum_{k=1}^{n} c_{k}(t)+r_{n}(t)
$$

The process from step 1 to step 6 is the complete procedure of EMD. The IMFs, $c(t)$, generated by EMD can be further processed to generate analytic forms of mode functions by Hilbert trandform. The amplitude and instantaneous frequency can then be derived via the analytic forms of mode functions. These calaulations can be expressed as follows:

$$
\begin{gathered}
z_{i}(t)=a_{i}(t) \cdot e^{j \theta_{i}(t)} \\
a_{i}(t)=\sqrt{c_{i}(t)^{2}+y_{i}(t)^{2}} \\
\theta_{i}(t)=\tan ^{-1} \frac{y_{i}(t)}{x_{i}(t)} \\
w_{i}(t)=\dot{\theta}_{i}(t)
\end{gathered}
$$

where $z_{i}(t)$ is the analystic form of $\operatorname{IMF}_{i}$ and $y_{i}(t)$ is the image part of IMF $i$ derived by Hilbert transform, $a_{i}(t)$ is the amplitude of IMF $i, \theta_{i}(t)$ is the phase of IMF $i$, and $w_{i}(t)$ is the instantaneous frequency of IMF $i$.

Furthermore, the kinetic energy of each IMF is defined as the square of amplitude, and the frequency of each IMF is defined as the average of instantaneous frequency. The kinetic energy is used to present the energy of a special component, which represents the energy of a parasympathetic or sumpathetic tone. An ANS has sympathetic and parasympathetic components. In modulation frequencies of the heart rate, sympathetic activity is associated with the LF while parasympathetic activity is associated with HF $[10,11]$. In this paper, the frequency of IMF is used to check the frequency bands of LF, HF, or very low frequency (VLF) to verifify the correlated mechanisms of an ANS, which the particular IMF reflects. 


\section{METHODS}

\section{ECG holter}

In this study, an ECG holter developed by Micro-Star International (MSI) (instrument model of MyECG E-380 [12]) was used to record ECG signals. Since it was a threelead ECG holter, four electrodes are required for three measuring points and the ground. These four electrodes are noted as left arm (LA), right arm (RA), left leg (LL), and right leg (RL). The placement of electrodes is shown in Figure 2. In an ECG holter, the sampling rate can be set at 250,500 , or $1000 \mathrm{~Hz}$. In this investigation, the sampling rate was $500 \mathrm{~Hz}$. Traditionally, ECG signal is composed of PQRST waves (P wave represents atrial depolarization; QRS complex represents ventricular depolarization; and $\mathrm{T}$ wave reflects the rapid repolarization of ventricles) and each $\mathrm{R}$ peak must be filtered to evaluate the HRV of the whole surgery. This ECG holter provides an automatic function for $\mathrm{R}$-wave detection. The noise types were electromyographic interference, $60 \mathrm{~Hz}$ power line interference, baseline drift due to respiration, abrupt baseline shift, and a composite noise constructed from all of the other noise types. Fortunately, the MSI ECG holter filtered out these noises and sent each normal-to-normal interval for offline analysis. They were then transferred to heart rate time series with a re-sampling rate of $2 \mathrm{~Hz}$. Before further analysis, the heart rate time series were decomposed into the first 8 IMFs. The frequency of each IMF was derived using the average of instantaneous frequency and compared to the frequency bands of ANS to figure out its corresponding frequency band, as shown in Table 1. According to Table 1, IMF 2 reflects the component of the HF band and IMFs 3-4 reflect the component of the LF band.

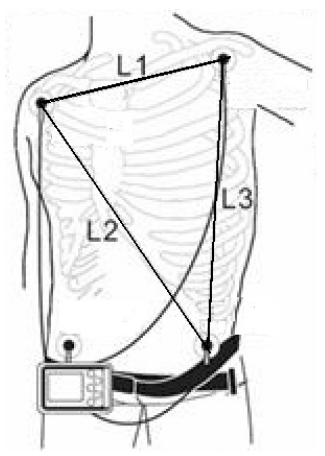

Figure 2. Illustration of electrode placements for MSI ECG holter [9]

Table 1. Averaged frequency for each IMF decomposed from human heart rate time series

\begin{tabular}{ccc}
\hline IMF & Frequency $(\mathbf{H z})$ & Corresponding frequency band \\
\hline IMF1 & 0.53 & \\
IMF2 & 0.24 & HF \\
IMF3 & 0.11 & LF \\
IMF4 & 0.05 & LF \\
IMF5 & 0.02 & VLF \\
IMF6 & 0.01 & VLF \\
IMF7 & 0.004 & VLF \\
IMF8 & 0.002 & VLF
\end{tabular}




\section{Subjects:}

This study was approved by the Research Ethics Board of National Taiwan University Hospital and an informed consent was obtained from every participant. The inclusion criteria were gestational age of 36 weeks or above, singleton pregnancy, no significant medical or obstetric complications, and no evidence of fetal or maternal compromise during the preceding period of labor. The exclusion criteria were inadequate analgesia (VAS $>3$ ), poor parturient compliance, gestational diabetic mellitus and mothers with cardiovascular diseases.

Based on the data of a previous study [14], which used 28 subjects (13 patients after heart transplantation for the experimental group, and 15 patients with severe heart disease for the control group) to access the sympathetic activation before and after transplantation by power spectral analysis of heart rate variability, we conducted a power analysis using the means, standard deviations, $\alpha$ and $\beta$ values of the experimental and control groups to calculate the expected group sizes for this study. The required minimum of satisfactory number was 12 . Thus, 13 pregnant women were recruited in the experimental group and delivered under continuous epidural bupivacaine $0.075 \%$ infusion with the height of epidural block located in the range from T7 to T8. ECG recording started when participants arrived at the hospital. The normal-to-normal intervals (NN-interval) were downloaded from an MSI ECG holter. Another 20 pregnant women with an average gestational age of 38.6 weeks were in the control group. The basic information of the experimental and control groups is shown in Table 2.

Table 2. Basic information for the experimental and control groups. Data are shown in (means \pm standard deviation)

\begin{tabular}{ccc}
\hline & Control group & Experimental group \\
\hline $\mathbf{n}$ & $\mathbf{2 0}$ & $\mathbf{1 3}$ \\
\hline Age $(\mathrm{yr})$ & $30.15 \pm 2.66$ & $30.38 \pm 3.27$ \\
Weight $(\mathrm{Kg})$ & $65.20 \pm 4.76$ & $62.69 \pm 4.46$ \\
BMI $\left(\mathrm{Kg} / \mathrm{m}^{2}\right)$ & $25.61 \pm 1.52$ & $24.68 \pm 1.71$ \\
Gestational age $(\mathrm{wk})$ & $38.6 \pm 1.10$ & $38.62 \pm 1.04$
\end{tabular}

\section{Statistical analysis}

Values were expressed as (means \pm S.D). Because the sample size is too small to form normal distributions, a non-parametric statistical method was employed. Data obtained from the two groups (viz. control and experimental groups) were compared by KruskalWallis test [13], and $\mathrm{P}<0.05$ was considered statistically significant.

\section{RESULTS}

In this investigation, the duration of the shift time-window was 2 hours in EMD. Figure 3 shows a set of IMFs decomposed from heart rate time series from a subject in the experimental group. In order to demonstrate how the anaesthetic affects the ANS, a sample of the continuous change of energy of a subject in the experimental group is shown in Figure 4. Also, in order to compare the ANS activities between the experi- 


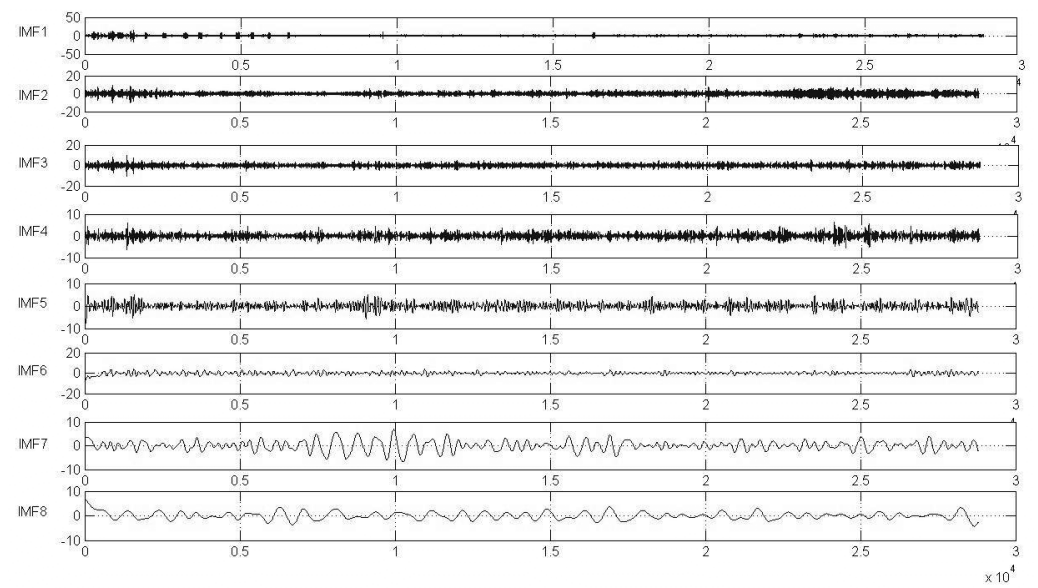

Figure 3. The first 8 IMFs decomposed from human heart rate time series of a subject in the experimental group.
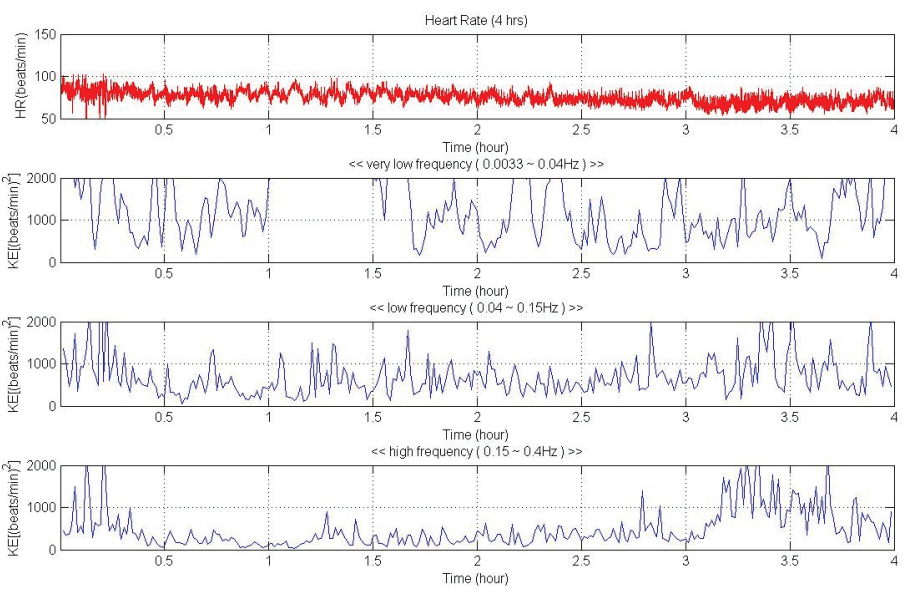

Figure 4 The kinetic energy of the corresponding IMFs to HF and LF of ANS for a subject in the experimental group [unit: (beats/min $)^{2}$ ]

mental and the control groups, a sample of the continuous change of energy of a subject in the control group is shown in Figure 5. Comparison of Figures 4 and 5 shows that the change of energy in control subjects without anesthesia is more active (greater change of energy) than experimental subjects receiving continuous epidural analgesia. Data of average energy of the total continuous HF are exhibited in Table 3 for the subjects in the control group, and in Table 4 for the experimental group. It is shown that the average energy of HF for the control group is greater than the experimental group ( $p=$ 0.008). Data of the average energy of the total continuous LF are shown in Table 5 for 

on the Autonomic Nervous System Using Hilbert Huang Transform
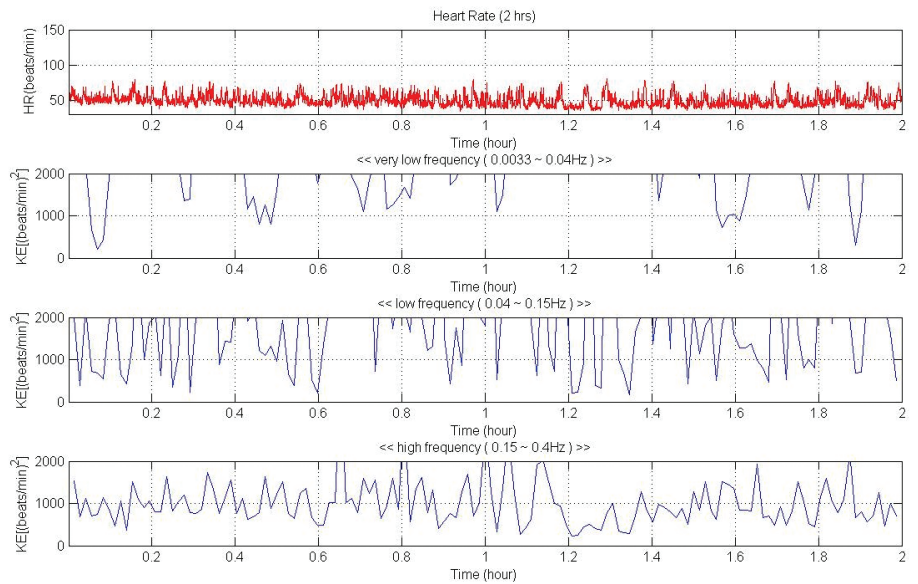

Figure 5. The kinetic energy of the corresponding IMFs to HF and LF of ANS for a subject in the control group [unit: (beats/min)2]

Table 3. Average energy of HF for the control group

\begin{tabular}{cc}
\hline Subject number & $\begin{array}{c}\text { Average energy of HF } \\
\text { (beats } / \text { min) })^{2} \text { in logarithm }\end{array}$ \\
\hline 1 & 5.893 \\
2 & 6.918 \\
3 & 5.971 \\
4 & 5.871 \\
5 & 7.657 \\
6 & 5.253 \\
7 & 5.240 \\
8 & 6.348 \\
9 & 6.936 \\
10 & 5.506 \\
11 & 5.936 \\
12 & 6.322 \\
13 & 4.009 \\
14 & 4.806 \\
15 & 5.276 \\
16 & 4.200 \\
17 & 6.625 \\
18 & 6.937 \\
19 & 7.337 \\
20 & 5.772 \\
\hline Mean & 5.941 \\
\hline Standard deviation & 0.981 \\
\hline
\end{tabular}


Table 4. Average energy of HF for the experimental group

\begin{tabular}{cc}
\hline Patient number & $\begin{array}{c}\text { Average energy of HF } \\
\text { (beats/min) } \text { ' }^{\text {in logarithm }}\end{array}$ \\
\hline 1 & 6.169 \\
2 & 5.523 \\
3 & 4.837 \\
4 & 4.943 \\
5 & 5.248 \\
6 & 4.263 \\
7 & 3.428 \\
8 & 4.924 \\
9 & 4.029 \\
10 & 5.561 \\
11 & 5.229 \\
12 & 6.096 \\
13 & 3.223 \\
\hline Mean & 4.882 \\
\hline Standard deviation & 0.924 \\
\hline
\end{tabular}

Table 5. Average energy of LF for the control group

\begin{tabular}{cc}
\hline subject number & $\begin{array}{c}\text { Average energy of LF } \\
\text { (beats/min) }{ }^{2} \text { in logarithm }\end{array}$ \\
\hline 1 & 7.188 \\
2 & 7.753 \\
3 & 6.964 \\
4 & 7.517 \\
5 & 7.150 \\
6 & 6.629 \\
7 & 6.163 \\
8 & 7.539 \\
9 & 7.539 \\
10 & 6.976 \\
11 & 6.864 \\
12 & 6.986 \\
13 & 4.309 \\
14 & 6.360 \\
15 & 6.429 \\
16 & 6.278 \\
17 & 6.616 \\
18 & 6.987 \\
19 & 7.470 \\
20 & 6.801 \\
\hline Mean & 6.826 \\
\hline Standard deviation & 0.748 \\
\hline &
\end{tabular}



on the Autonomic Nervous System Using Hilbert Huang Transform

Table 6. Average energy of LF for the experimental group

\begin{tabular}{cc}
\hline Patient number & $\begin{array}{c}\text { Average energy of } \mathbf{L F} \\
\text { (beats/min) })^{\mathbf{2}} \text { in logarithm }\end{array}$ \\
\hline 1 & 6.550 \\
2 & 6.360 \\
3 & 6.433 \\
4 & 6.495 \\
5 & 5.546 \\
6 & 6.228 \\
7 & 6.319 \\
8 & 6.563 \\
9 & 6.139 \\
10 & 7.450 \\
11 & 6.524 \\
12 & 6.609 \\
13 & 6.158 \\
\hline Mean & 6.414 \\
\hline Standard deviation & 0.419 \\
\hline
\end{tabular}

Table 7. Average energy ratio of $\mathrm{LF} / \mathrm{HF}$ for the control group.

\begin{tabular}{|c|c|}
\hline Volunteer number & Average energy ratio of $\mathrm{LF} / \mathrm{HF}$ \\
\hline 1 & 3.65 \\
\hline 2 & 2.30 \\
\hline 3 & 2.70 \\
\hline 4 & 5.19 \\
\hline 5 & 0.60 \\
\hline 6 & 3.96 \\
\hline 7 & 2.52 \\
\hline 8 & 3.29 \\
\hline 9 & 1.83 \\
\hline 10 & 4.35 \\
\hline 11 & 2.53 \\
\hline 12 & 1.94 \\
\hline 13 & 1.35 \\
\hline 14 & 4.73 \\
\hline 15 & 3.17 \\
\hline 16 & 7.99 \\
\hline 17 & 0.99 \\
\hline 18 & 1.05 \\
\hline 19 & 1.14 \\
\hline 20 & 2.80 \\
\hline Mean & 2.90 \\
\hline Standard deviation & 1.76 \\
\hline
\end{tabular}


Table 8. Average energy ratio of $\mathrm{LF} / \mathrm{HF}$ for the experimental group

\begin{tabular}{cc}
\hline Patient number & Average energy ratio of $\mathbf{L F} / \mathbf{H F}$ \\
\hline 1 & 1.46 \\
2 & 2.31 \\
3 & 4.93 \\
4 & 4.72 \\
5 & 1.35 \\
6 & 7.14 \\
7 & 18.02 \\
8 & 5.15 \\
9 & 8.25 \\
10 & 6.61 \\
11 & 3.65 \\
12 & 1.67 \\
13 & 18.83 \\
\hline Mean & 6.47 \\
\hline Standard deviation & 5.75 \\
\hline
\end{tabular}

subjects in the control group, and in Table 6 for the experimental group. It is shown that the average energy of LF for the control group is greater than the experimental group ( $p=0.007)$. Further, the data of the average energy ratio of LF / HF in Table 7 for the control group and Table 8 for the experimental group demonstrate a higher value for the experimental group than the control group $(p=0.033)$.

\section{DISCUSSION}

The general belief is that the activity of parasympathetic tone is lower than the main functional activity of other major systems in the human body. However, our result indicates that the high frequency heart rate under the effect of parasympathetic tone was lowered by the anesthesia. According to the established theory, the result should have been an increase in heart beat, which in turn increases blood flow. However, a previous study [1] showed that continuous epidural bupivacaine $0.075 \%$ infusion increases the resistance of uterine artery and therefore possibly reduces the uterine blood flow. One possible explanation for this observation is that the balance of the ANS was disrupted. Therefore, when the parasympathetic tone was broken by epidural bupivacaine, the sympathetic tone was also affected at the same time. The interaction between sympathetic and parasympathetic is well known as an important mechanism of ANS.

According to previous studies [15-17], the low/high frequency energy ratio reflects the index of sympathy-vagal balance, which means the sympathetic and parasympathetic components of ANS are all suppressed by continuous low-dose epidural bupivacaine $0.075 \%$ infusion, but the parasympathetic power is suppressed more than the sympathetic power. Our results agree with the previous studies.

In future research, we will attempt to obtain longer ECG signal records in order to observe full heart rate variations before and after continuous epidural bupivacaine 
$0.075 \%$ infusion. We speculate that the onset of labor also influences heart rate variation and deserves investigation using HHT method to obtain a suitable EMD. The resulting ECG from lying-in women who do not undergo painless labor would be processed to eliminate the effect of labor on heart rate variation. These results can be compared with those undergoing painless labor in order to better understand the relation between autonomic nervous system and heart rate variation.

The present work suggests that HHT may be a valuable tool to investigate ANS response. It may be worthwhile to compare the performances of the traditional methods (such as that employed in Akselrod et al. [3]) and the HHT-based method in evaluating ANS responses.

\section{CONCLUSIONS}

In this paper, it was found that the change of energy in pregnant women in labor without anesthesia is more active than those receiving continuous epidural analgesia via bupivacaine $0.075 \%$ infusion. The energy values of HF and LF ANS activities were significantly lower in subjects receiving analgesia than those without, but the trend for energy ratio of $\mathrm{LF} / \mathrm{HF}$ was the opposite. The current results suggest that the sympathetic and parasympathetic components of ANS were all suppressed by continuous low-dose epidural bupivacaine $0.075 \%$ infusion, but the parasympathetic power was suppressed more than the sympathetic power. However, due to the interactive counteraction of the automatic nervous system, this result would be affected when the electricity releasing rate from the parasympathetic nerve is affected. Therefore, the automatic nervous system would be affected under continuous low-dose epidural bupivacaine infusion during painless delivery.

\section{ACKNOWLEDGEMENTS}

The authors wish to thank the National Science Council (NSC) of Taiwan (Grant Number: NSC96-2221-E-155-015-MY3) for supporting this research.

\section{REFERENCES}

[1] L. K. Chen, C. J. Lin, C. H. Huang, M. H. Wang, P. L. Lin, C. N. Lee and W. Z. Sun, "The effects of continuous epidural analgesia on Doppler velocimetry of uterine arteries during different periods of labor analgesia", British Journal of Anaesthesia 96, p.226-230, 2006.

[2] S. Akselrod, D. Gordon, F.A. Ubel, D.C. Shannonet, A.C. Barger and R.J. Cohen, "Power spectrum analysis of heart rate fluctuation: a quantitative probe of beat-to-beat cardiovascular control," Science, vol. 213, no. 10, p.220-222, 1981.

[3] A. Lindqvist, "Noninvasive methods to study autonomic nervous control of circulation," Acta Physiological Scandinavica, Suppl. vol. 588, pp.1-107, 1990.

[4] R Introna, E Yodlowski, J Pruett, N Montano, A Porta, R Crumrine. Sympathovagal effects of spinal anesthesia assessed by heart rate variability analysis. Anesth Analg 80:315-321, 1995.

[5] A Deschamps, I Kaufman, S.B. Backman, G Plourde. Autonomic nervous system response to epidural analgesia in laboring patients by wavelet transform of heart rate and blood pressure variability. Anesthesiology 101:21-27, 2004.

[6] D.P. Landry, F.M. Bennett, N.E. Oriol. Analysis of heart rate dynamics as a measure of autonomic tone in obstetrical patients undergoing epidural or spinal anesthesia. Reg Anesth 19:189-195, 1994. 
[7] JT Bigger, Jr, JL Fleiss, RC Stemman, LM Rolnizky, RE Kleiger and JN Rottman, ”Frequency domain measures of heart period variability and mortality after myocardial infarction", Circulation 85, p.164$171,1992$.

[8] N.E. Huang, Z. Shen, S.R. Long, M.C. Wu, H.H. Shih, Q.Zheng, N.C. Yen, C.C. Tung and H.H. Liu, "The empirical mode decomposition and the Hilbert Spectrum for nonlinear and nonstationary time series analysis", Proceedings of Royal Society of London, Series A 454, p.903-995, 1998.

[9] E. Bedrosian, "A product theorem for Hilbert transform.” Proc. IEEE 51, 868-869, 1963.

[10] M.F. Abbod, J. S. Shieh, J. R. Yeh, K. Y. Cheng, S.J. Huang, Y.Y. Han, "Intelligent Systems for the Prediction of Brain Death Index", IEEE Biocas2008 conference, November 20 - 22, Baltimore, USA , 2008.

[11] J.S. Shieh, M.F. Abbod, J.R. Yeh, K.Y. Cheng, S.J. Huang, Y.Y. Han, "Hard and soft computing applied to prediction of brain-death patients categories in neurosurgical intensive care units", In: Artificial Intelligence: New Research, R.B. Bernstein and W.N. Curtis (Ed.), Nova Science Publishers, pp. 379403, 2009.

[12] The operation manual of MYECG E-380, publication of Micro-Star International, 2007.

[13] W.H. Kruskal and W.A. Wallis," Use of Ranks in One-Criterion Variance analysis", Journal of the American Statistical Association, 47:583-621, 1952.

[14] A. Mortara, M. T. La Rovere, M. G. Signorini, P. Pantaleo, G. Pinna, L. Martinelli, C. Ceconi, S. Cerutti, and L. Tavazzi," Can power spectral analysis of heart rate variability identify a high risk subgroup of congestive heart failure patients with excessive sympathetic activation? A pilot study before and after heart transplantation," Br. Heart Journal, 71(5)::422-430, 1994.

[15] B. Pomeranz, R.J.B. Macaulay, M.A. Caudill, I. Kutz, D. Adam, D. Gordon, K.M. Kilborn, A.C. Barger, D.C. Shannon, R.J. Cohen, H. Benson, "Assessment of autonomic function in humans by heart rate spectral analysis", Am. J. Physiol. Heart Circ. Physiol. 248, H151-H153, 1985.

[16] M. Pagani, F. Lombardi, S. Guzzetti, O. Rimoldi, R. Furlan, P. Pizzinelli, G. Sandrone, G. Malfatto, S. Dell'Orto, E. Piccaluga, "Power spectral analysis of heart rate and arterial pressure variabilities as a marker of sympatho-vagal interaction in man and conscious dog", Circ. Res. 59, 178-193, 1986.

[17] R.G. Yeh, J.S. Shieh, G.Y. Chen, D.D. Kuo, "Detrended fluctuation analysis of short-term heart rate variability in late pregnant women", Autonomic Neuroscience-Basic \& Clinical, 2009 (article in press). 



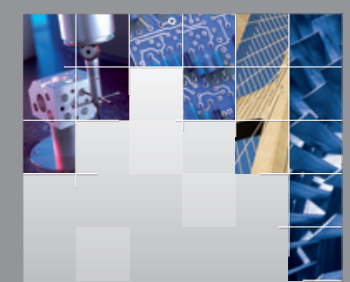

\section{Enfincering}
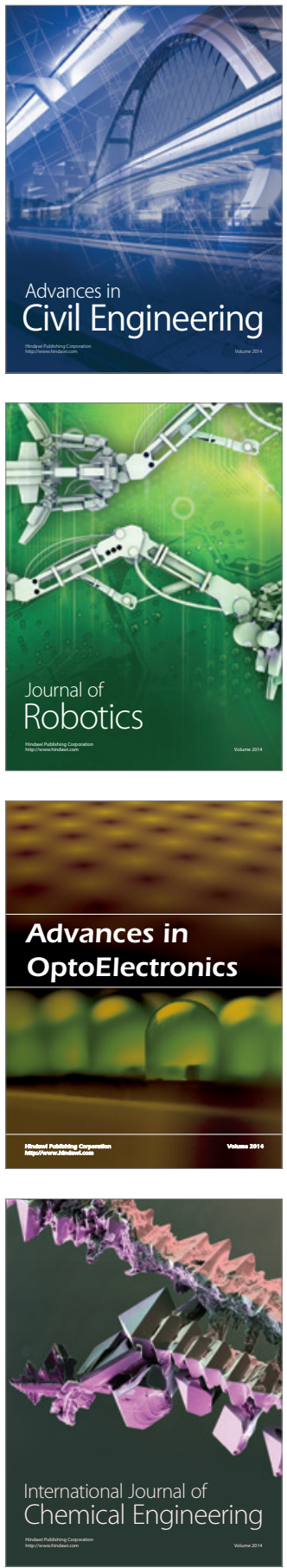

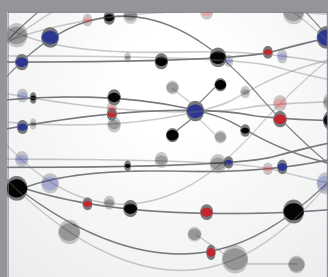

The Scientific World Journal

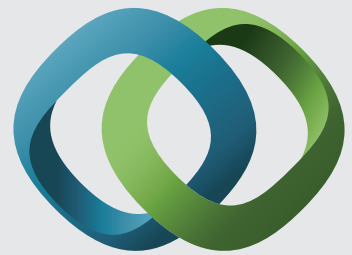

\section{Hindawi}

Submit your manuscripts at

http://www.hindawi.com
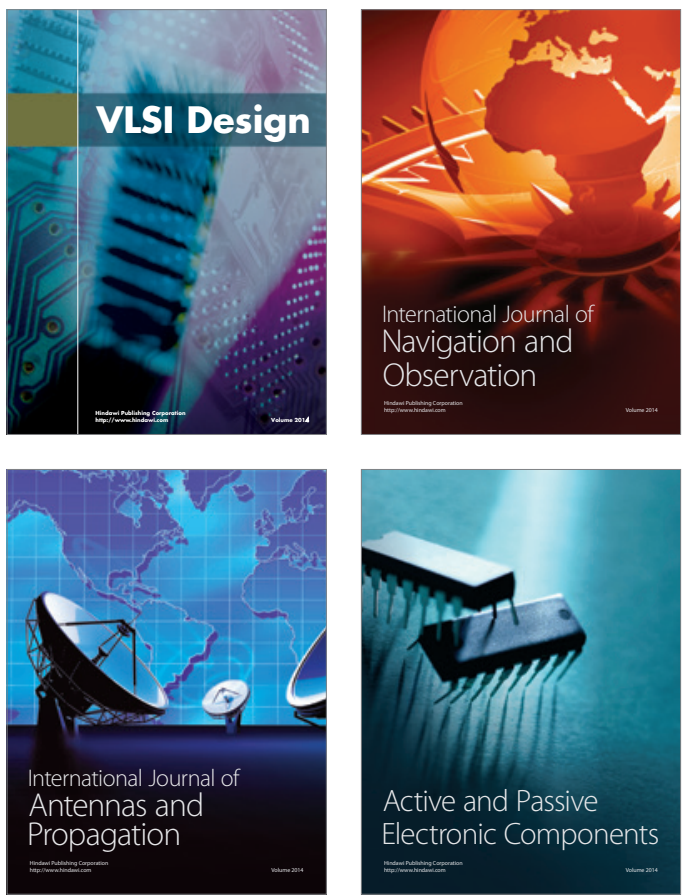
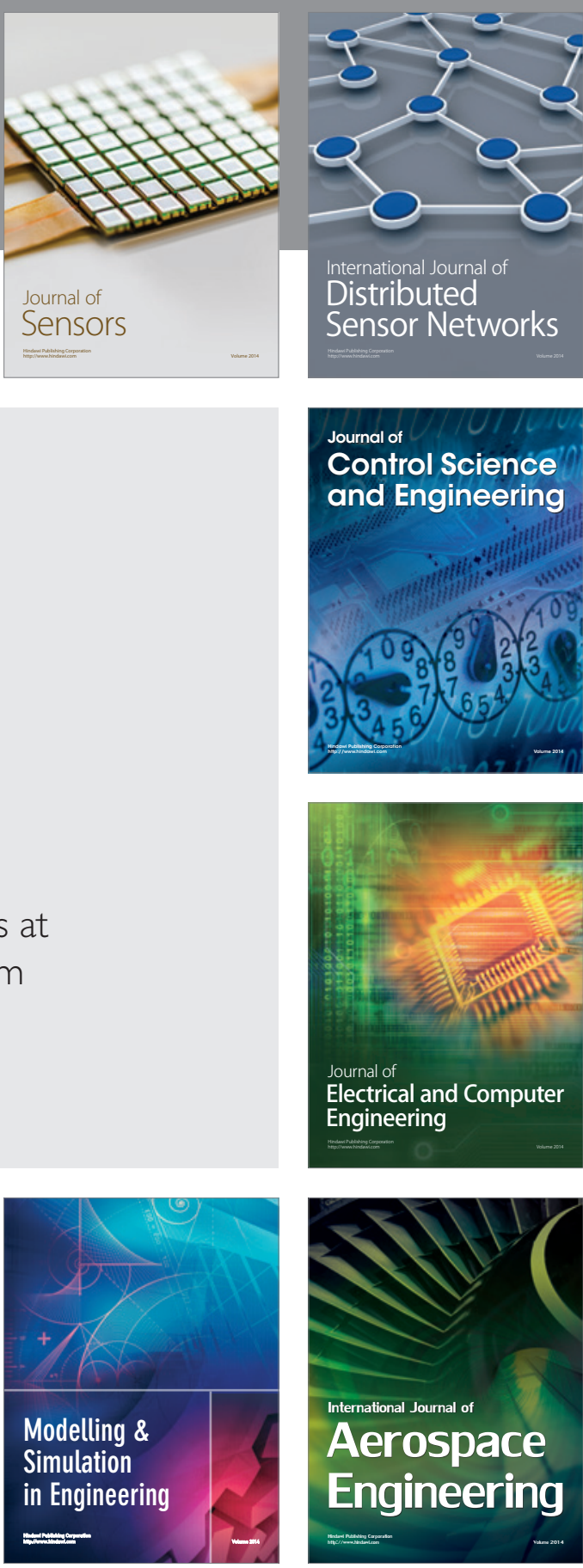

International Journal of

Distributed

Sensor Networks

Journal of

Control Science

and Engineering
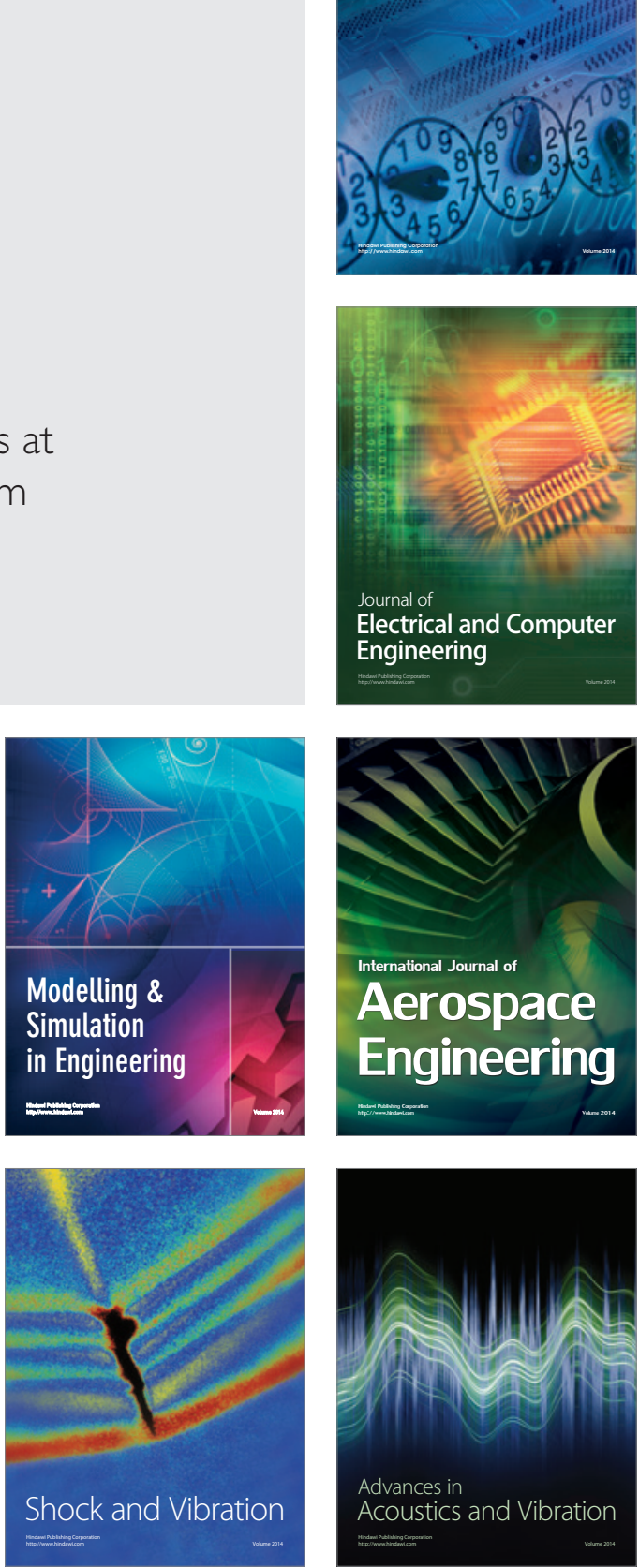